\title{
A Study on the Relationship between the Marine Experience Factors and the Satisfaction Index of the Northeast Asian Sea Region in the PKNU Maritime Index
}

\section{Minho Yang}

Pukyong National University, Institute for Humanities and Social Sciences, Korea

minhoyang@pknu.ac.kr
Joonhwan Kim

Sungkyul University, Department of Paideia, Korea

kjh715@sungkyul.ac.kr

Publication Information:

Received 19 October 2020, Accepted 30 November 2020, Available online 22 December 2020

DOI: 10.21463/jmic.2020.09.2.11

\section{Abstract}

This study used the experience satisfaction index from the PKNU Maritime Index to examine Koreans' preference for marine experience and use the findings as basic data for policymaking in the future. The PKNU Maritime Index of Koreans was 585.3 out of 1,000, and the experience satisfaction index was 63.6. By focusing on the experience satisfaction index, this study confirmed that the PKNU Maritime Index had changed dynamically. The analysis showed that compared to the sub-domains of the PKNU Maritime Index, the degree of experience satisfaction had continued to increase after the introduction of the index. This paper further studied the correlation and influence between the marine experience factors and the satisfaction index. Above all, this study's results are expected to provide practical implications for establishing policy alternatives related to marine experience in the Northeast Asian sea region and be used as basic data for future research.

Keywords

PKNU Maritime Index, Experience Satisfaction Index, Marine Experience Factors, Regression Analysis 


\section{Introduction}

With relatively longer coastlines than its land area, three seas with unique coastlines, over 3,000 small and large islands, and the world's five largest mudflats, South Korea has excellent marine tourism resources. Moreover, with the rise in income levels and more leisure time, and as travel trends increasingly value unique experiences, the marine tourism industry has become an industry to be fostered continuously through the development of contents that could be enjoyed at the sea and policy support for facility development (Son, 2010). However, the national tourism policy has been based on land-based resources despite the marine tourism industry's high potential. Policy for fostering marine experience tourism, on the other hand, has focused on creating facilities and infrastructure. Despite the increase in the number of people enjoying marine leisure sports, support for this sector has been lacking and ended in one-time events. Previous research on marine leisure sports activities also had limits in its legal and policy foundation. However, the Korean government has recently established a framework to invigorate marine tourism by forming a liaison between the Ministry of Culture, Sports and Tourism, which is in charge of tourism, and the Ministry of Oceans and Fisheries, which oversees marine policy, to collaborate on establishing effective marine tourism policies and implementing them more comprehensively (Ministry of Oceans and Fisheries, 2018). However, economic activities have stagnated due to the recent COVID-19 "pandemic." As people have become more reluctant to share spaces, marine leisure tourism is facing a crisis. The global COVID-19 pandemic is ongoing, and the global lifestyle has transformed into a "contactless" lifestyle, significantly affecting the health of human beings and businesses and national economies worldwide. Political, social, and economic activities have transformed into digital forms by becoming contactless and going online. As physical experiences have transitioned into online experiences, new contactless trends that employ digital technology have appeared. Such changes suggest that the consumers' perception toward the marine experience industry and their interest and demand can be improved by breaking away from the traditional way of delivering marine tourism-related content through knowledge or stories. Instead, the industry could provide consumers with realistic marine tourism content by using augmented reality (AR) or virtual reality (VR) and actively interacting with marine tourism content (Jung \& Song, 2017).

Research on experience could be classified into studies that focused on the experiential aspects of processing information (Holbrook \& Hirschman, 1982), information economics studies that classify goods or attributes by the point of purchase when the consumer obtains the information (Nelson, 1970), the experience economy perspective that emphasizes the experience of pleasure in the evolution of goods in accordance with the stages of economic development (Pine \& Gilmore, 1998) and the view that emphasizes the total experience by classifying all consumer responses as experiences, as presented by Schmitt (1999). As mentioned above, many researchers have different views toward experience research. Pine and Gilmore posits that the experience economy to be a transformation of a single economic concept and argued that the first stage of the economy was centered on commodities, the second stage on goods, the third on service, and the fourth stage on experience (Pine \& Gilmore, 1998). In this sense, the experience economy is a concept that encompasses the foundations of the current economy while creating additional added values. The experience economy also defines authentic experiences as an upper concept of goods and services and especially considers the consumer experience of directly participating in the production process to define the authentic experience. In this regard, Chang (2017) examines the experience economy index based on the experienced utility theory and analyzes the travel patterns, perception, and behavior by groups in accordance with the experience economy index. 
Pukyong National University has also developed evaluation items and methods for developing marine humanities and has objectively measured Koreans' awareness of the sea every year since 2017. The "2019 PKNU Maritime Index" showed that the "familiarity index" and "experience satisfaction index" were each 70.6 and 70, maintaining similar levels as the previous year (Yang \& Choi, 2020). This showed that Koreans had maintained relatively higher levels of familiarity and satisfaction toward the sea. However, previous studies have focused on tourism and festivals by researching marine tourism resources, methods to foster marine tourism, marine cultural festivals, and marine tourism satisfaction, rather than conducting a comprehensive analysis of the marine culture or systematic research on marine experience (Hyun, Rui \& Lee, 2019). Consumers nowadays do not only value high-quality experience and service but seek self-realization through symbolic and self-expressive experiences. Consumers seek psychological satisfaction and demand differentiated experience and service. By using the experience satisfaction index, this paper analyzed what values consumers seek through marine experience and how marine experience activities and marine cultural activities affect satisfaction levels, hoping that the experience satisfaction index can be used for comprehensive research on marine experience in the future. In addition, to revitalize marine experience in the Northeast Asian sea region, this study proposed a systematic and integrated approach to marine experience through continuous measurement of the experience satisfaction index, which could be used as basic data for research to identify factors for providing marine experience opportunities.

\section{Relevant Work}

\subsection{PKNU Maritime Index}

Various surveys and research have focused on the meaning of the sea. However, most have been limited to specific institutions or topics, while research on the meaning of the sea in a marine humanities perspective has been severely lacking. Pukyong National University created the PKNU Maritime Index by conducting a survey in 2017 and 2018 and asking "what the sea means to Koreans." In 2019, researchers added more items to understand the Korean awareness of the Korean sea and their humanistic significance (Yang \& Choi, 2020).

The PKNU Maritime Index identifies Koreans' perception of the sea region and the humanities of the sea region and understands the sea region perception and thinking according to the experiences of regions, generations, and individuals. As of October 2019, the sample frame was the number of people registered in the resident registration demographics of Ministry of the Interior and Safety. For the research method, a 1:1 face-to-face interview was conducted, and a structured questionnaire was used for data collection. The collected data (raw data) was statistically processed with the SPSS program after editing and coding. The survey results were analyzed after weighting by reflecting the population distribution by region, sex, and age. Also, the '100-point average $(Y n=(X n-1) \star 100 / 4)^{\prime}$ in some statistical tables is the average value obtained by converting the response value $(\mathrm{Xn})$ of the scale item into a 100-point score. Table 1 presents the contents of the sub-index. 
Table 1. Sub-index of PKNU Maritime Index

\begin{tabular}{|c|c|}
\hline Dimension & Description \\
\hline Familiarity Index & The degree to which you feel positive about the sea \\
\hline Knowledge Index & Level of knowledge about the sea \\
\hline Experience Satisfaction Index & Ocean-related activities and cultural experiences, frequency, and satisfaction \\
\hline Safety Index & $\begin{array}{l}\text { Whether you are familiar with direct/indirect experiences of marine safety accidents, common sense about safety, and } \\
\text { behavior }\end{array}$ \\
\hline Security Index & Degree of securing maritime sovereignty and territorial sovereignty and maritime security \\
\hline Food Index & Intake frequency, satisfaction, preference, recommendation intention of sea food \\
\hline Environment Index & Satisfaction with the marine environment, personal perception and behavior of the marine environment \\
\hline Economy Index & Impact of the sea on individual, regional, national economies \\
\hline Education Index & Marine education level, satisfaction, policy will in various educational institutions \\
\hline Policy Index & Importance, effectiveness, and policy compliance of maritime policy \\
\hline $\begin{array}{l}\text { Northeast Asian Sea Region } \\
\text { Index }\end{array}$ & The degree of influence of neighboring countries in the Northeast Asian sea region \\
\hline
\end{tabular}

During the first two years, the PKNU Maritime Index consisted of the familiarity index, food index, marine culture index, security and safety index, environment index, and knowledge index. The scores for each index were calculated on a 100point basis and then converted based on 1,000 points, and there were differences in the scores for each index. This survey showed that the "familiarity index" and "experience satisfaction index" were each 64.1 and 63.6, which were similar or higher than that of 2018 (72.6 and 69.9 respectively), showing that the Koreans' level of familiarity and satisfaction toward the sea were maintained at a high level. On the other hand, the security index dropped from 60.9 in 2017 to 56.2 in 2018 and 54.6 in 2019. The policy index also plummeted as the 2018 score of 74.1 dropped to 53.1, indicating that the public's perception of maritime security has changed negatively. The safety index has also fallen from 50.8 points in 2018 to 47.4 points in 2019 as $61.7 \%$ of the respondents responded "yes" to the item "Education programs are needed to reinforce marine safety consciousness." and only $38.2 \%$ replied positively to the item "I am well aware of the initial response methods in case of a marine safety accident."

The Northeast Asian Sea Region index, which was newly instated in 2019, was a relatively low 51.1 . Only $29.2 \%$ of the respondents replied that they were "aware" of the item "I am aware of the history of overseas Koreans living in China and Japan." For the item "The ship routes in modern Korea, China, and Japan affected the establishment of relations between the three countries," only $37.4 \%$ replied that "the ship routes had an influence." The free association survey conducted along with the marine index survey showed that the Korean public considered Busan harbor to be the representative harbor in Korea. The respondents further answered that Jeju island was the most representative as an island, the Haeundae beach as a beach, and mackerel as the most representative type of fish. The free association survey results confirmed that the sea's public "image" has remained unchanged for three years. 


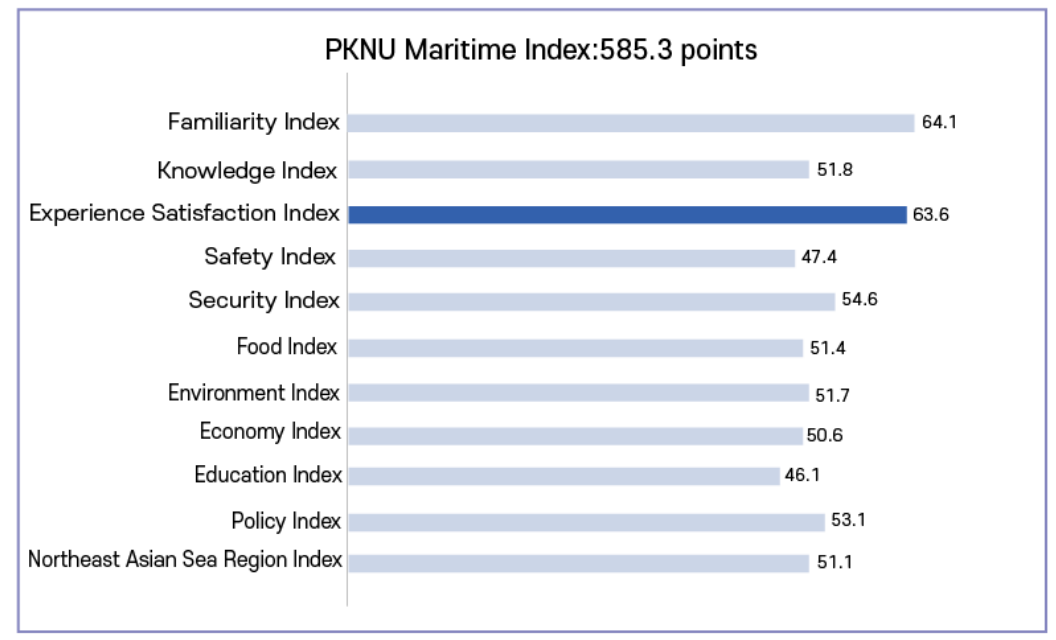

Fig 1. PKNU Maritime Index

\subsection{Experience Satisfaction Index}

The experience satisfaction index was measured through the experience, frequency, and degree of satisfaction with marine activities or cultural experiences. This study also examined the respondents' satisfaction with marine leisure and cultural activities they experienced during the past year and their perspectives on the prospects of existing marine experience and culture.

The experience satisfaction index, which indicated the degree of satisfaction with marine experience activities, was 63.6. Questions on the experience, frequency, and degree of satisfaction with various marine experience activities showed that the rates of experiencing marine tourism experience (70.3\%) and acquiring maritime information through media (68.8\%) were high. The frequency of activities was 1 to 3 times in the past year in most activities, and the satisfaction level for all activities was high, with an average of 60 points or higher.

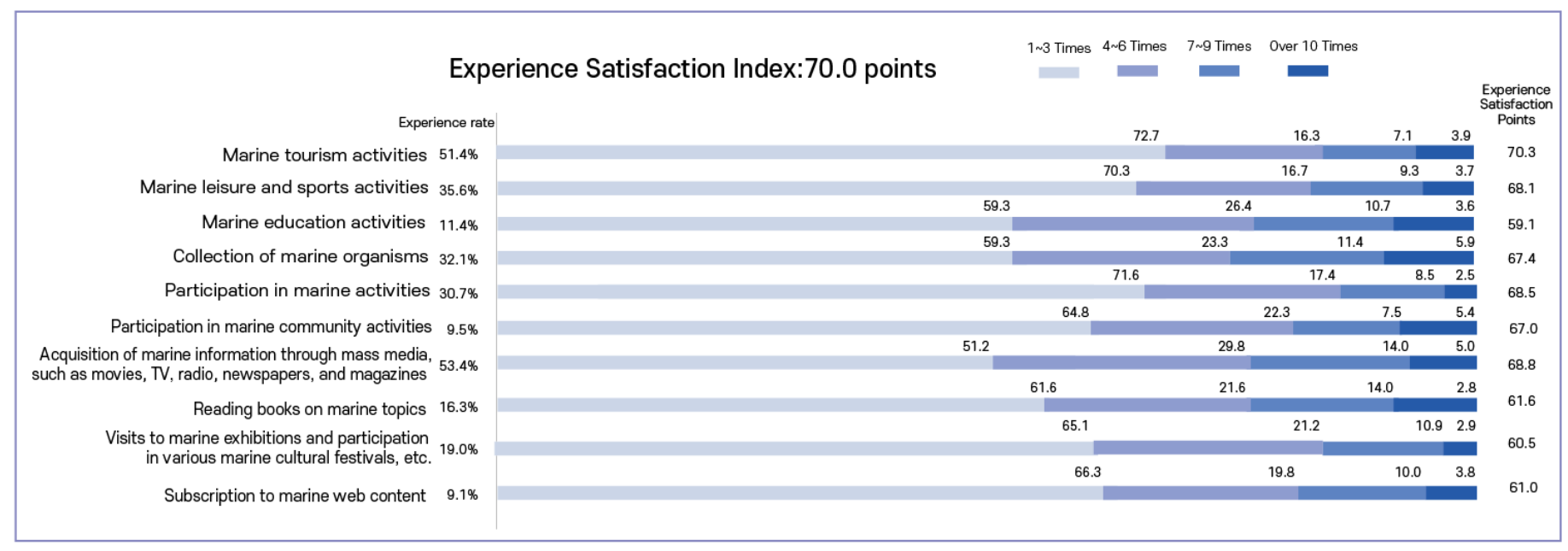

Fig 2. Experience Satisfaction Index 


\subsection{Trend Analysis of the PKNU Maritime Index and the Experience Satisfaction Index}

First, trends of the PKNU Maritime Index and the experience satisfaction index are shown in Figure 3 and Figure 4. Overall, the experience satisfaction index score started at a higher level than the PKNU Maritime Index. The PKNU Maritime Index began with 62.6 points in 2017 when it was first introduced, then reached 59.8 points in 2018 and 58.5 as of 2019. The scores fluctuated in general, displaying an irregular pattern. The experience satisfaction index began as a marine cultural index in 2017 with 70.0, reached 69.9 points in 2018 and 63.6 in 2019. Overall, there was little fluctuation. Among the sub-domains of the PKNU Maritime Index, the familiarity index (70.6) and the experience satisfaction index (70.0) was relatively higher, while the safety index (52.1) and the education index (50.7) was considerably lower. The scores for each index were calculated on a 100-point basis and then converted based on 1,000 points, and there were differences in the scores for each index.

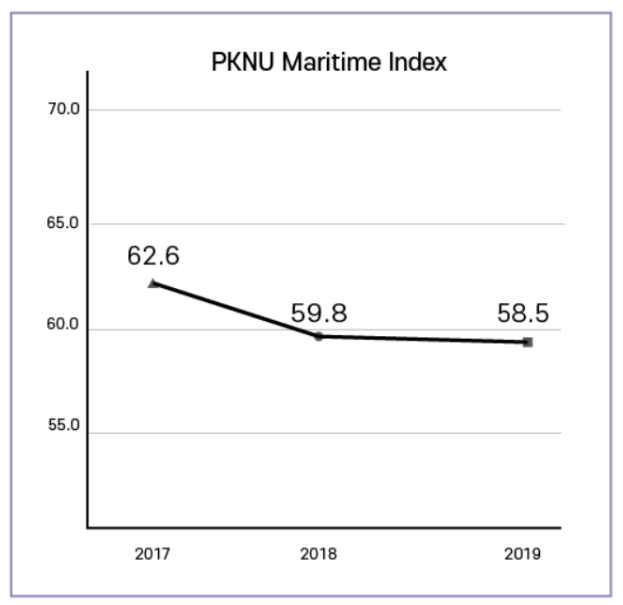

Fig 3. Rates of Increase of PKNU Maritime Index

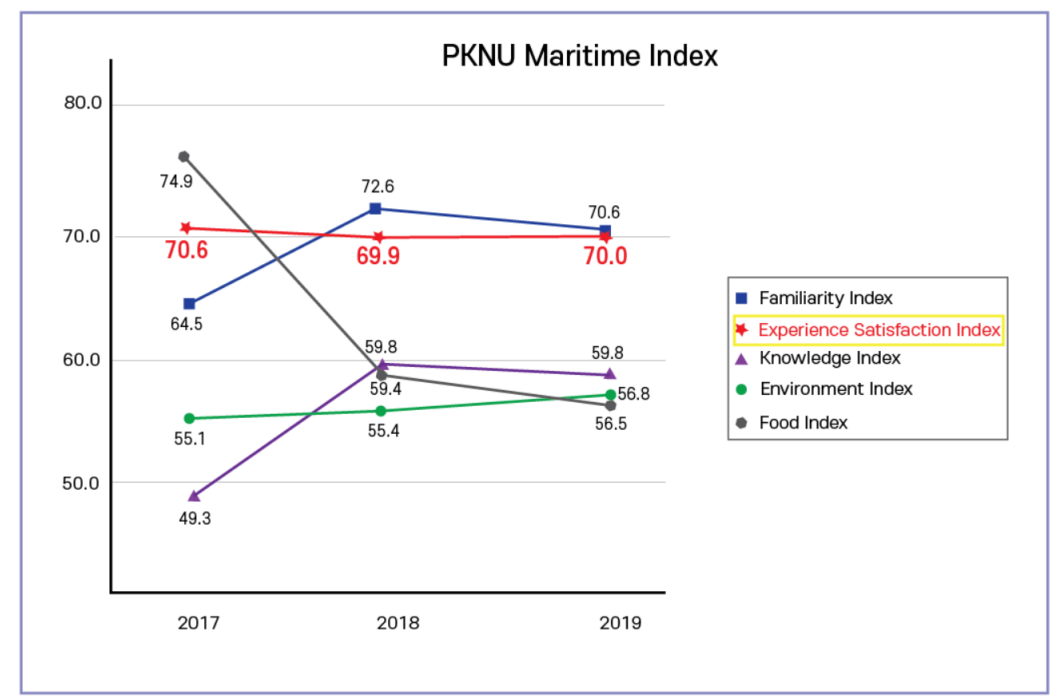

Fig 4. Rates of Increase of Experience Satisfaction Index 


\subsection{Satisfaction Level of marine Leisure and Cultural Activities by Year}

An analysis of marine leisure and cultural activities' satisfaction rates showed that the item "The marine leisure/cultural activities were a pleasant experience." had the highest score of 64.9. On the other hand, the item "The cost of marine leisure/cultural activities is not a waste at all." scored a lower score of 59.9.

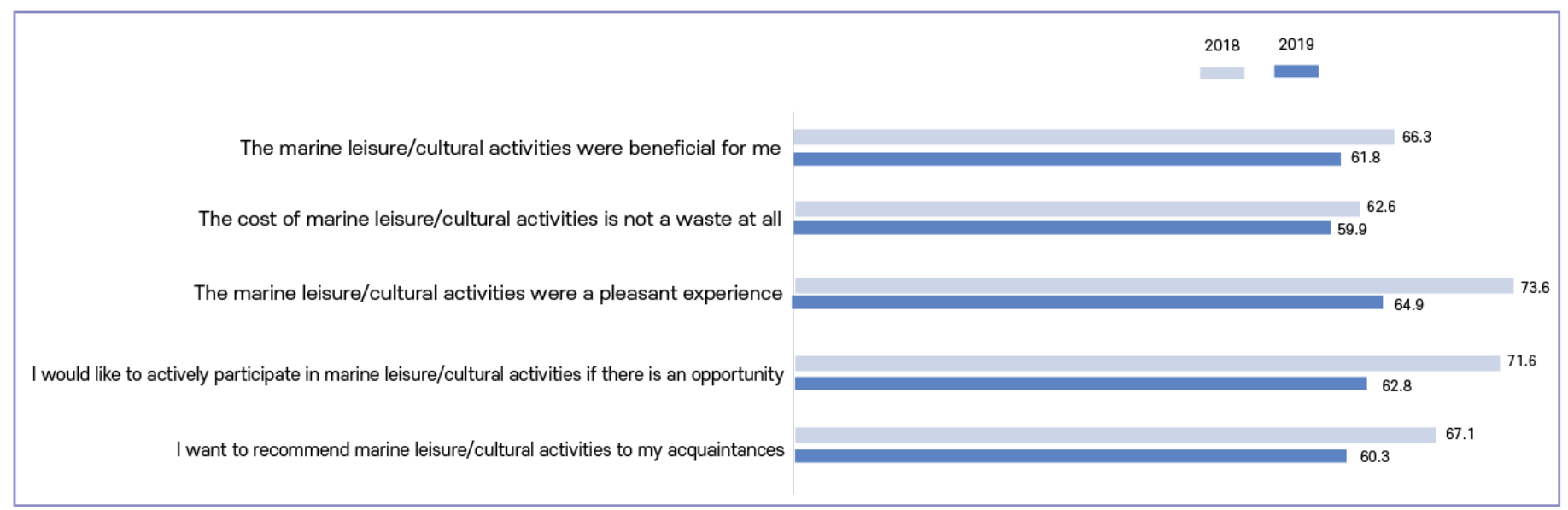

Fig 5. Satisfaction Level of Marine Leisure and Cultural Activities by Year

\subsection{The Possibility of Transmitting Marine Experience and Culture}

Regarding the possibility of marine experience and culture being passed down, over half of the respondents replied that "they will be passed down" (66.7\%). The possibility of whether the marine experience and culture could be transmitted varied by region. The number was measured higher in the Busan region (88.8) than the rest.

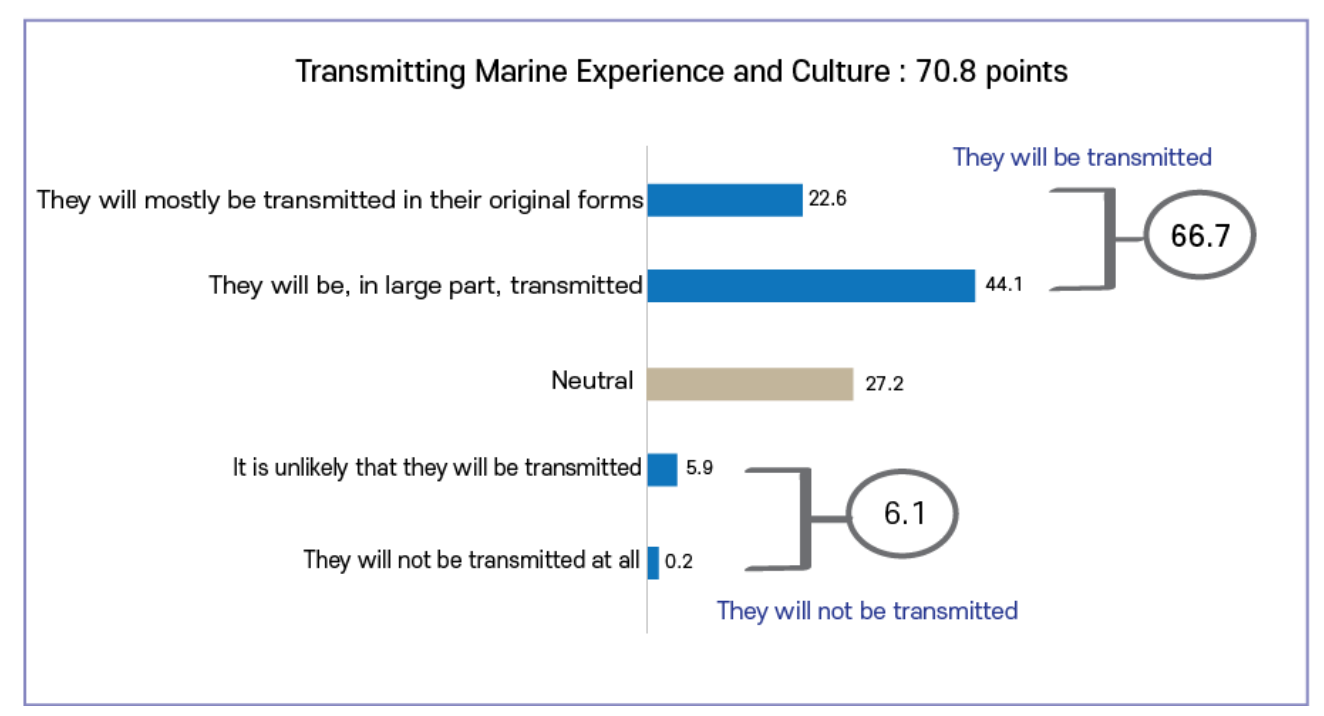

Fig 6. The Possibility of Transmitting Marine Experience and Culture 


\section{Study Design and Methods}

\subsection{Sample and Data Collection}

To verify the results, this study analyzed the relation between the experience satisfaction index (including marine experience activities and marine cultural activities) from the PKNU Maritime Index, which was measured by the Pukyong University Humanities Korea Plus Project Team, and the level of satisfaction (PKNU Maritime Index, 2019). The research subjects included in the analysis were 1,200 adult men and women nationwide whose responses were collected through a 1:1 face-to-face interview survey from October 14 to October 23, 2019. The research subjects were classified according to demographic characteristics for statistical analysis (Table 2). The questionnaire was constructed on a Likert 5-point scale, and the hypotheses were tested using correlation analysis and regression analysis with SPSS 24.0.

Table 2. Respondents' Demographic Profiles

\begin{tabular}{|c|c|c|c|}
\hline \multicolumn{2}{|c|}{ Variables } & \multirow[t]{2}{*}{ Frequency } & \multirow{2}{*}{$\frac{\%}{50.0}$} \\
\hline Gender & Male & & \\
\hline & Female & 600 & 50.0 \\
\hline \multirow[t]{5}{*}{ Age } & 29 and below & 219 & 18.3 \\
\hline & $30-39$ & 216 & 18.0 \\
\hline & $40-49$ & 238 & 19.8 \\
\hline & $50-59$ & 244 & 20.3 \\
\hline & 60 and above & 283 & 23.6 \\
\hline \multirow[t]{8}{*}{ Region } & Seoul / Gyeonggi & 260 & 21.7 \\
\hline & Incheon & 75 & 6.3 \\
\hline & Pusan & 80 & 6.7 \\
\hline & Chungcheong & 224 & 18.7 \\
\hline & Jeonra & 183 & 15.3 \\
\hline & Gyeongsang & 273 & 22.8 \\
\hline & Gangwon & 59 & 4.9 \\
\hline & Jeju & 46 & 3.8 \\
\hline \multirow[t]{4}{*}{ Marine related work / Interest } & Yes & 67 & 5.6 \\
\hline & No / Yes & 643 & 53.6 \\
\hline & No / Yes & 489 & 40.8 \\
\hline & No Response & 1 & 0.1 \\
\hline \multirow[t]{3}{*}{ Living experience near the sea } & No & 694 & 57.8 \\
\hline & Past & 148 & 12.3 \\
\hline & Current & 358 & 29.8 \\
\hline
\end{tabular}




\subsection{Research Hypotheses}

Experience research has been covered in various fields, including marketing, consumer behavior, and psychology. However, existing experience research has been centered on the type and importance of experience (Kim \& Jung, 2018), and not many studies have approached experience activities using indices (Chang, 2017). Experience could be defined as what one sees, hears, or goes through, or the process or the knowledge or skills gained in the process. It could also refer to the subjective consciousness one gains through their senses or introspection. Such a definition of experience is "comprehensive in that it encompasses the action and process itself and also the outcome of the learning experience." In other words, the experience is an integrated power that kindles human sensibility and reason through sense and introspection.

Schmitt (2010) argued that "experiences are responses triggered by specific stimuli, and are private events that occur to individuals. Such experiences are psychological responses that encompass both ration and emotion of human beings." In some ways, it is related to the technology that affects most experiences, and in another, it is related to the intensity of market competition that forces differentiation. Therefore, various efforts are being made to improve customer satisfaction and product value by providing a high level of experience. Previous studies have shown that the experience factor affects consumer satisfaction (Lim \& Lee, 2019).

In particularly, Kim (2019) confirmed in a study on tourists who experienced marine tourism activities that the marine tourism experience factor positively affected on the level of satisfaction. Previous research also found that the experience factor of participants of the Korea International Marine Leisure Week (KIMA WEEK) yacht experience positively affected the experience satisfaction (Kim, Jung \& Chung, 2018).

Moreover, Han, Lee and Choi (2014) found that among the experience factors experienced by people participating in marine leisure sports, the escapist experience factor had a more significant effect on experience satisfaction. Studies also suggest that experiences that stimulate emotions could affect the customers' feelings and emotions and lead to a positive attitude toward services (Hollbrook \& Batra, 1987). Based on existing research and reasoning, this study posited the following hypotheses.

Hypothesis 1: The marine activity experience will positively affect the satisfaction of leisure and cultural activities. Hypothesis 2: The marine culture experience will positively affect the satisfaction of leisure and cultural activities.

\subsection{Analysis and Results}

A regression analysis was conducted to test the relationship between the experience factors of the experience satisfaction index (marine activity experience and marine culture experience) as independent variables and the satisfaction level of marine leisure and cultural activities as dependent variables. First, the descriptive statistics and correlation coefficients of each variable in this study can be found in Table 3 . The correlations between the variables were all significant at the $p<.001$ level. The correlation between the marine activity experience and the satisfaction level $(r=.445, p<.001)$, the marine culture experience and the satisfaction level $(r=.407, p<.001)$, and the marine activity experience and the marine culture experience $(r=.795, \mathrm{p}<.001)$ displayed significant positive correlations. A multicollinearity issue can occur if the correlation between the variables is 0.8 or higher. However, the tolerance was all .100 or higher, and the VIF was less than .10, which made the data suitable for the regression model. 
The regression analysis for identifying whether the factors of marine experience activities and marine culture activities affect the satisfaction levels of marine leisure and cultural activities showed that both of the marine experience activities and the marine culture activities had significant influences on the satisfaction levels of leisure and cultural activities (Table 4). The predictors of the regression model explained $17.7 \%$ of the variances in the satisfaction levels.

Table 3. Descriptive Statistics and Correlations

\begin{tabular}{|l|l|l|l|l|l|}
\hline \multicolumn{1}{|c|}{ Variables } & \multicolumn{1}{|c|}{ Mean } & \multicolumn{1}{|c|}{ SD } & \multicolumn{1}{|c|}{$\mathbf{1}$} & \multicolumn{1}{|c|}{$\mathbf{2}$} & \multicolumn{1}{|c|}{} \\
\hline The marine activity experience & 3.4974 & .8590 & $(.909)$ & & \\
\hline The marine culture experience & 3.3784 & .9803 & $.795^{\star \star}$ & $(.897)$ & \\
\hline The satisfaction of leisure and cultural activities & 3.4895 & .6750 & $.445^{\star \star}$ & $.407^{\star \star}$ & $(.868)$ \\
\hline
\end{tabular}

Note. ${ }^{\star \star} p<.001$. Numbers in parentheses are Cronbach's alpha coefficients.

Table 4. Results of Regression Analysis

\begin{tabular}{|l|l|l|l|l|l|}
\hline \multicolumn{1}{|c|}{ Variables } & \multicolumn{1}{c|}{$\mathrm{B}$} & \multicolumn{1}{c|}{$\boldsymbol{\beta}$} & \multicolumn{1}{c|}{$\mathrm{SE}$} & \multicolumn{1}{c|}{$\mathrm{t}$} & \multicolumn{1}{c|}{ VIF } \\
\hline The marine activity experience & .445 & .381 & .098 & $3.880^{\star *}$ & 1.000 \\
\hline The marine culture experience & .407 & .299 & .079 & $3.786^{\star *}$ & 1.000 \\
\hline $\begin{array}{l}\text { Dependent variable: The satisfaction of leisure and cultural activities } \\
\mathrm{F}=15.055^{\star *}, \mathrm{R}^{2}=.198, \text { Adjusted } \mathrm{R}^{2}=.185\end{array}$ & \\
\hline
\end{tabular}

Note. ${ }^{\star \star} \mathrm{p}<.001$

\section{Discussion}

This study is significant as a comprehensive marine awareness survey that started from a humanistic consideration of what kind of sea and marine culture will be passed down to the next generation and used the PKNU Maritime Index, an index that comprehensively reflects the perception of the sea by the Korean public. The empirical analysis was also conducted to identify the relationship between the experience satisfaction index (experience factor) and satisfaction level. This paper also presented ideas to vitalize marine experience by verifying the correlation between these two variables and how they affect each other. The key implications of this study were as follows.

Through a national-level survey, this study researched what the "sea" means to Koreans by region, generation, and individual experience, which could be used as basic data for future research in marine humanities, marine education, and marine culture industry. More specifically, this study used the compiled data on the experience satisfaction index to empirically check whether there were specific patterns in the dynamic movement of the index. First, the experience satisfaction index was confirmed to have increased over time. This pattern was found in both the PKNU Maritime Index and the experience satisfaction index, showing that from a marine humanistic perspective, Koreans have an accurate definition of the "sea." The annual release of the PKNU Maritime Index is expected to contribute to the rise in marine experience as more relevant institutions, businesses, and consumers utilize the results. 
Second, this study analyzed and applied the relationship between the experience satisfaction index and the satisfaction level using the PKNU Maritime index. The spread of COVID-19 is aggravating the crisis in the marine tourism and leisure industry worldwide. The rise of the fear of face-to-face contact has led to a change in the society's overall perception and behavior of a new "contactless" lifestyle, which is a new way of consumption that avoids any interpersonal or face-toface contact. The dramatic rise in the demand for contactless services and the acceleration of the digital economy could be used as an opportunity for future growth. More specifically, marine experience strategies should be established and redesigned by capturing the change in consumer trends in the new reality brought about by COVID-19. For example, consumers will be able to participate in and obtain information on their desired experience anytime and anywhere without being impeded by place or time through new technologies that have been spreading recently, such as VR and AR. Even after the end of the COVID-19 pandemic, consumers will continue to demand the comfort and speed of contactless services. Contactless experiences would continue to be developed to meet such consumer demands. The recent AR technology has been combined with the offline, online, or mobile platform to provide consumers with the information on goods more effectively and interactively (Jung, Yeo \& Yoon, 2018; Vonkeman, Verhagen \& Dolen, 2017). Since AR and VR technologies are being applied in various fields, strategies to apply AR and VR technology in marine experience activities are necessary.

While this study has various implications by analyzing the experience satisfaction index, there were some limitations and directions for future research. The analysis conducted in this study has the limitation of not being more elaborate due to the lack of data on the existing items related to various experiences. Future studies may utilize the items based on Pine and Gilmore (1998)'s theory of experience economy, which are classified into four different aspects or the measurement tool using five experiential factors - sense, feel, think, act, and relate - presented by Schmitt (1999). Future studies should further use such measurement tools to examine how the marine experience environment could be managed using new technology such as VR and AR. In addition, the subject of this survey was limited to Koreans, and the study did not encompass the Northeast Asian sea region, including China and Japan. Since there would be limitations to individual policy studies in understanding the impact of the experience satisfaction index in the Northeast Asian sea area, a more comprehensive perspective on marine experience and strategies is necessary.

\section{Acknowledgments}

This work was supported by the Ministry of Education of the Republic of Korea and the National Research Foundation of Korea (NRF-2017S1A6A3A01079869).

\section{References}

Chang, S. H., 2017. Perceptual and Behavioral Differences among Experience Economy Value-Based Groups: Focused on Deajeon Metropolitan Destination Visitors. Journal of Tourism Sciences, 41(3): 85-104.

Han, T. Y., Lee, K. S., Choi, S. B., 2014. The relationships to study of experiential service value and satisfaction and intentions to among marine sport loyalty of Gangwon province East Sea beach visitor. Korean Journal of Sports Science, 23(3): 103-113.

Holbrook, M. B., Batra, R., 1987. Assessing the role of emotions as mediators of consumer responses to advertising. Journal of Consumer Research, 14(3): 404-420. 
Holbrook, M. B., Hirschman, E. C., 1982. The experiential aspects of consumption: consumer fantasies, feelings, and fun. Journal of Consumer Research, 9(2): 132-140.

Hyun, M., Rui, D. G., Lee, C. S., 2019. A Study on maritime situation through marine culture index and Korean wave synergy. Journal of Korea Research Association of International Commerce, 19(1): 47-68.

Jung, Y. S., Yeo, C. K., Yoon, S. C., 2018. A study on the development of marine leisure industry based on experience marketing: Focusing on virtual experience technology. Journal of Tourism Studies, 30(2): 33-54.

Jung, Y. S., Song, I. K., 2017. A study on the flow and expansion of augmented reality and virtual reality in distribution industry: Focused on major success cases. Journal of Distribution and Management Research, 20(5): 23-34.

Kim, C. H., Jung, Y. S., 2018. A study on the effects of experiential marketing on satisfaction, loyalty and purchase intention : Differences between user groups in marine leisure industry Korean. Journal of Business Administration, 31(3): 603-624.

Kim, C. W., Jung, Y. S., Chung, W. S., 2018. The structural relationship between experience factor, experience satisfaction and re-participation intention of KIMAWEEK yacht experience participants. Journal of the Korea Contents Association, 18(5): 409-419.

Kim, Y. K., 2019. A study on effects among experience factors, experience satisfaction and behavioral intention of marine tourism participants. Journal of Tourism and Leisure Research, 31(1): 75-91.

Lim, M. J., Lee, S. H., 2019. The effect of marine sports event experience on visitor`s satisfaction and behavior intention. Journal of Product Research, 37(3): 121-127.

Nelson, P., 1970. Information and consumer behavior. Journal of Political Economy, 78(2): 311-329.

Ministry of Oceans and Fisheries, 2018. Ministry of Culture, Sports and Tourism, Ministry of Oceans and Fisheries set out to revitalize marine tourism. Press release, 2018. 7. 11.

Pine, B. J., Gilmore, J. H., 1998. Welcome to the experience economy. Harvard Business Review, 76: 97-105.

Pukyong University, 2019. PKNU Maritime Index Survey on the Northeast Asian Sea Region. Pukyong University HK+ Research Group.

Schmitt, B. H., 1999. Experiential marketing. Journal of Marketing Management, 15(1-3): 53-67.

Schmitt, B. H., 2010. Customer experience management: A revolutionary approach to connecting with your customers. John Wiley \& Sons.

Son, M. S., 2010. In the era of 30,000 dollars in income, the marine tourism and leisure industry is leading. Nara Economy, 9: 68-69.

Vonkeman, C., Verhagen, T., van Dolen, W., 2017. Role of local presence in online impulse buying. Information \& Management, 54(8): 10381048.

Yang. M. H., Choi, M. K., 2020. A sociolinguistic study on the consciousness of humanities in Northeast Asian Sea Region?- Focus on 'Maritime Cultural Index'. Institute for Humanities and Social Sciences, 21(1): 27-52. 